\title{
A Novel 4D Track Prediction Approach Combining Empirical Mode Decomposition with Nonlinear Correlation Coefficient
}

\section{Un nuevo enfoque de predicción de vías en 4D que combina la descomposición modal empírica con el coeficiente de correlación no lineal}

DOI: $10.46932 / \mathrm{sfjdv2n4-019}$

Received in: March 1st, 2021

Accepted in: May 30th, 2021

\section{Zhiyuan Shen}

$\mathrm{Ph} . \mathrm{D}$

College of Civil Aviation, Nanjing University of Aeronautics and Astronautics, Nanjing, China Building A08, Nanjing University of Aeronautics and Astronautics No 29 JiangJunDaDao, Jiangning

District, Nanjing, China, 210016

shenzy@nuaa.edu.cn

\section{Xiaowei Wang}

Master Student

College of Civil Aviation, Nanjing University of Aeronautics and Astronautics, Nanjing, China Building A08, Nanjing University of Aeronautics and Astronautics No 29 JiangJunDaDao, Jiangning District, Nanjing, China, 210016

103256355@qq.com

\author{
Qiuxiang Wu \\ Master Degree \\ Nanjing LES information technology Co. LTD \\ No 8 Yongzhi St,Qinghuai District, Nanjing China \\ 28804332@qq.com
}

\begin{abstract}
The accuracy of 4D track prediction plays an important role to solve the prominent contradiction between the rapid development of air transport industry and the limited resources of airspace. The conventional 4D track prediction based on the aerospace dynamic model is usually inaccurate since of weather influence and air traffic controller (ATC) factor. In this paper, an entirely data-driven nominal flight height profile prediction approach combing empirical mode decomposition (EMD) with nonlinear correlation coefficient (NCC) is proposed. Firstly, the historical tracks are implemented on EMD individually. Then according to a procedure similar to leave-one-out cross validation (LOOCV), the physical meanings of different intrinsic mode functions (IMFs) obtained by EMD are analyzed to corresponding to the various flight information. For a specified flight, the similarities between different dates are measured by NCC. Finally, a predicted nominal trajectory is obtained by summing a series of selected IMFs with a regression weight under least square optimization framework. It is demonstrated that the proposed method shows a higher prediction performance when comparing with the state of the art method named as nearest neighbor classification with dynamic time warping (DTW).
\end{abstract}

\section{RESUMEN}

La precisión de la predicción de la pista 4D desempeña un papel importante para resolver la importante contradicción entre el rápido desarrollo de la industria del transporte aéreo y los recursos limitados del espacio aéreo. La predicción convencional de la pista 4D basada en el modelo dinámico aeroespacial suele 
ser inexacta debido a la influencia de las condiciones meteorológicas y el factor del controlador de tráfico aéreo (ATC). En este trabajo, se propone un enfoque de predicción del perfil de altura de vuelo nominal totalmente basado en datos que combina la descomposición empírica de modos (EMD) con el coeficiente de correlación no lineal (NCC). En primer lugar, las pistas históricas se implementan en la EMD individualmente. A continuación, de acuerdo con un procedimiento similar al de la validación cruzada sin intervención (LOOCV), se analizan los significados físicos de las diferentes funciones de modo intrínseco (IMF) obtenidas por la EMD para que correspondan a las diversas informaciones de vuelo. Para un vuelo específico, se miden las similitudes entre las distintas fechas mediante NCC. Por último, se obtiene una trayectoria nominal predicha mediante la suma de una serie de FMI seleccionadas con un peso de regresión en el marco de la optimización de mínimos cuadrados. Se demuestra que el método propuesto muestra un mayor rendimiento de predicción en comparación con el método más avanzado denominado clasificación de vecinos más cercanos con deformación temporal dinámica (DTW).

\section{INTRODUCTION}

Air traffic flow is expected to increase rapidly over the coming decades [Gariel2011]. This increase leads to departure delays and en-route congestion. To address the challenge of additional safety and conflicts problems compared to the current situations, new technologies and procedures are being developed. To achieve this aim, FAA and Eurocontrol have planned a next generation air traffic management system which is based on 4D trajectory operation. In China, a plan for developing a new generation of air traffic management system is proposed by civil aviation administration of china (CAAC) in 2007, and it is expected to be completed in $2025^{[\mathrm{Lv} 2007]}$. This system carried by 4D trajectory management will improve the efficiency of air traffic and reduce pollution.

4D trajectory prediction is defined as a precise description of an aircraft path in 3D space and time. They usually divide a flight profile into several phases and intend to establish kinematics equations accurately. One of prevalent methods is using target windows (TWs) imposed at different parts of the

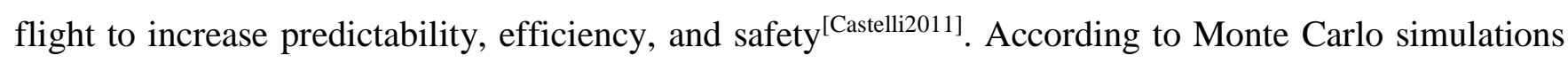
and reachability analysis, this method is extended to evaluate features of the TW concept ${ }^{[\text {Margellos2013] }}$. However, the uncertain factors likely as unpredicted weather, navigation error and aircraft modeling error usually degrade prediction accuracy. To break these constraints, track prediction is formalized as a stochastic optimization problem with generalized polynomial chaos ${ }^{\text {[Matsuno2013] }}$. Furthermore, Gariel carried out K-means clustering algorithm to analyze the trajectories in terminal area based on radar data but it is difficult to get the whole flight profile ${ }^{[\text {Gariel2011]. }}$

The previous mentioned 4D track prediction methods are usually constraint with the predetermined aerospace dynamic model. Therefore there is a growing interest in designing data-driven approach to improve the accuracy of trajectory prediction. One of core issues of these methods is nominal flight profile prediction based on historical flight data. To better mine flight intent hidden in data, a data- 
dependent adaptive framework is needed. As a nonlinear and non-stationary data analysis method, empirical mode decomposition (EMD) ${ }^{[\mathrm{Huang} 1998]}$ provides a facility to meet the above requirement. The effectiveness of this versatile time-frequency analysis method has been demonstrated by the fact that EMD is equivalent to a binary filter bank ${ }^{[\text {Flandrin2004] }}$.

According to EMD, a flight height/velocity profile can be decomposed into a collection of adaptive orthogonal components, called instinct mode functions (IMFs). To determine the physical meaning of each IMF corresponding to different flight intent, the correlation between the input flight data and sum function of selected IMFs is require to calculate. One of our previous proposed index, namely nonlinear correlation coefficient (NCC) ${ }^{[\text {Shen2009] }}$ is applied to achieve this aim.

The rest of this paper is organized as follows. In Section 2, the main concepts of EMD and NCC are briefly reviewed. In Section 3, the physical meaning of each IMF is analysis to predict flight height profile is obtained. Finally the conclusions are drawn in Section 4.

\section{REVEIWS OF METHODS}

\subsection{EMD ALGORITHM}

EMD is usually realized by considering the signal to be composed of a series of oscillation components. A function is called an IMF when it satisfies two conditions, namely: [i] in the whole signal, the number of extremes (maxima and minima) and the number of zero crossings must either be equal or differ at most by one; [ii] the mean value of the upper envelope corresponding to a local maximum and the lower envelope corresponding to a minimum must be zero $^{\text {[Huang2005]. }}$

According to EMD, any given input signal can be decomposed into a sequence of orthogonal IMFs in the following steps:

(1) Start with the given signal, set, and EMD sequence index to be 1;

(2) Fit the upper and lower envelopes of the input signal denoted as and, respectively.

(3) Subtract the mean envelop, from the input to get a residual signal. This procedure may be repeated several times to make sure the residual signal satisfies the definition of IMF.

(4) The resulting IMF is denoted as Increase to, check if the residual contains less than two extreme points. If not, go back to step 2.

Finally, the input signal is decomposed into IMFs and a residual as where denotes the residual after removing IMFs.

The time scale represented by each of the IMF is data-dependent because it is originated from the envelopes of the input signal. Furthermore, the time scale represented by different IMFs increases as . One reason is that the mean envelope at each is usually the slow changing component of the current 
signal. After it is subtracted from the input signal, the IMF represents the relative high-frequency component. While the final IMF, corresponds to the lowest frequency one and the residual is the trend of the signal and usually with strongest energy.

In numerical simulations, a maximum number of the IMFs is usually scaled to the logarithm of the length of input signal to prevent the appearance of over-sifting [Huang1999]. For instance, if the input signal is sampled into a vector, the number of the IMFs will typically be, denoting the maximum integer that is smaller than .

A historical flight height profile is implemented to EMD. The results are shown in Figure 1. From Figure 1, it is found that 5 IMFs labeled (a)-(e) corresponding to different flight intents are obtained. While the residual shown at the left bottom panels (f) of Figure 1 represents the overall flight trend including the factors as: taking off, cruising and landing.

Figure 1. EMD results of flight height data from NanChang to Xi'an (ZSCN-ZLXY) on Jan. 1st 2014
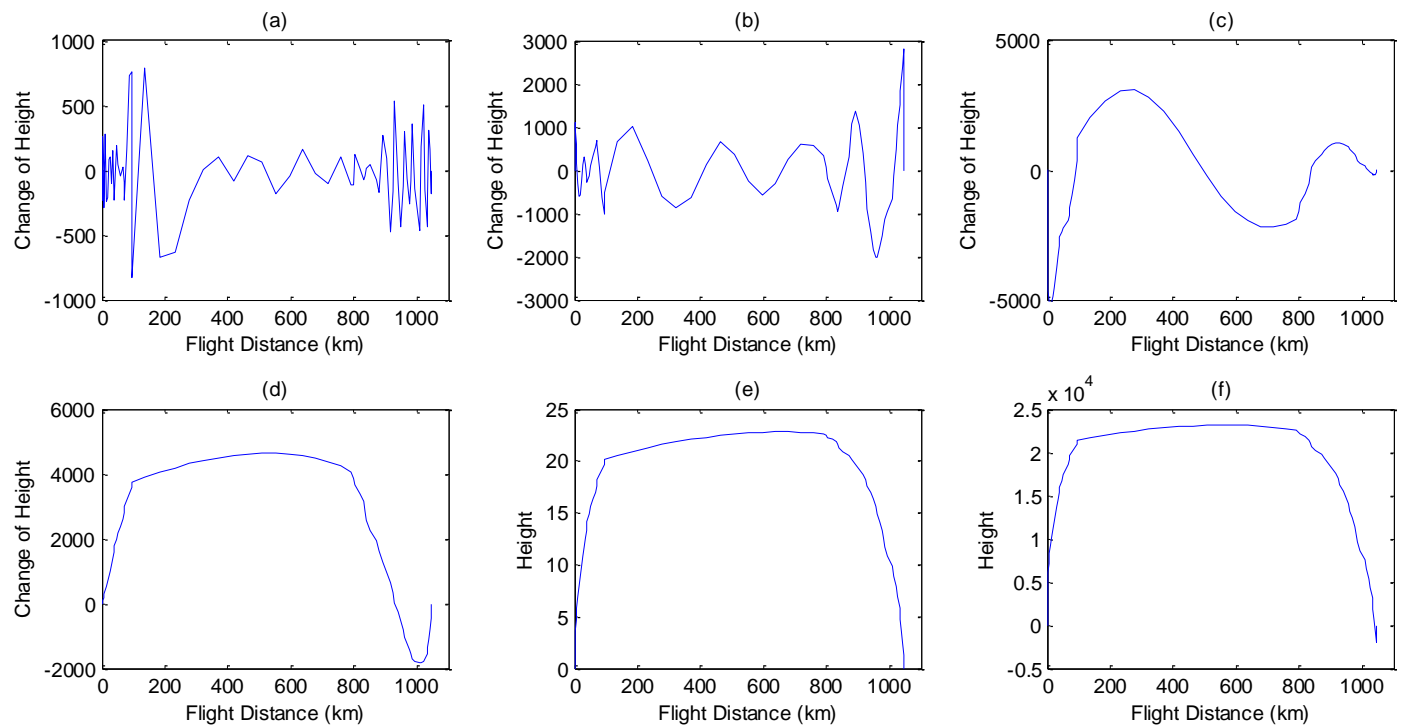

\subsection{NONLINEAR CORRELATION COEFFICIENT}

Multure information(MI) was a quantity to measure the mutual dependence of two variables, which is defined as NBwhere is the information entropy of the variable, and is the joint entropy of the two variables.

MI can be regarded as a generalized relationship analogous to the correlation, which is sensitive to any relationship, not just linear dependence. But its disadvantage is that it's not range in a closed interval, since .

To solve this problem, a revised version of the MI namely NCC based on statistical inference is proposed in our previous work ${ }^{[\mathrm{Shen} 2009]}$. Considering two discrete random variables and, they are first 
resorted in ascending order and placed into ranks with first samples in the first rank, the second samples in the second rank, and so on. Second, the sample pairs, , are placed into a rank grids by comparing the sample pairs to the rank sequences of and. The revised joint entropy of the two variables and is defined as where is the number of samples distributed in the rank grid. Then the nonlinear correlation coefficient is defined as where is the revised entropy of the variable

\section{NOMINAL FLIGHT HEIGHT PROFILE PREDICTION}

\subsection{PHYSICAL MEANING ANALYSIS OF IMFS}

To mine the representing flight intent for different IMFs, a similar procedure as leave-one-out cross validation (LOOCV) was applied. As it was shown in Figure 1, 5 IMFs were obtained when implementing EMD to flight height profile on specific day. The sum function of all IMFs expect the first one was calculated firstly as Then the sum of all IMFs without the second IMF was obtained as

This procedure was stopped until the sum function of all IMFs expects the last IMF was obtained. Finally, these five sum functions of the selected IMFs adding the residential component with the original input flight height curve were plotted in Figure 2.

Figure 2. Combination results of selected IMFs for NanChang to Xi'an (ZSCN-ZLXY) flight height profile on Jan. 1 st 2014

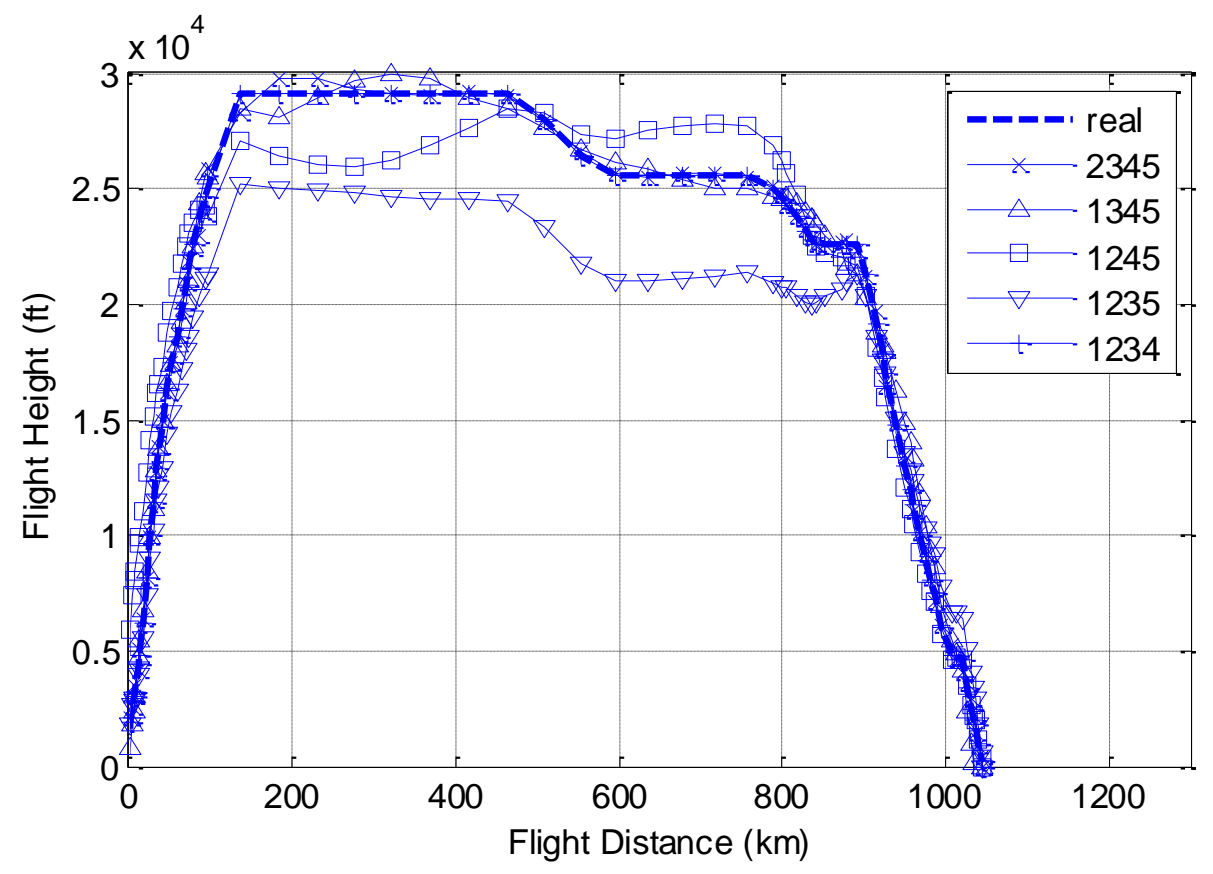

The EMD algorithm and above mentioned LOOCV procedure were also implemented to more flight height profiles on various days. The similar phenomena were observed. The NCC between the input 
data and sum functions of IMFs were calculated in Table 1. In the calculation of NCC, the parameter was set as 8 .

Table 1. NCC Results Between Original flight profile and the Sum Functions of Selected IMFs on Various Days

\begin{tabular}{l|l|l|l|l|l}
\hline & Day1 & Day2 & Day 3 & Day 4 & Day 5 \\
\hline & 0.9809 & 0.9144 & 0.9232 & 0.8960 & 0.9333 \\
& 0.9144 & 0.6519 & 0.7049 & 0.6813 & 0.8161 \\
& 0.6094 & 0.6102 & 0.6552 & 0.7340 & 0.6798 \\
& 0.7010 & 0.8019 & 0.8662 & 0.6190 & 0.6993 \\
& 0.9809 & 0.8525 & 0.9662 & 0.8557 & 0.9635 \\
\hline
\end{tabular}

Based on results shown in Figure 2 and Table 1, it was obviously found that the sum function of the first four IMFs can approximate the original flight height profile. It demonstrated that the last IMF is with no clear physical meanings. While the fourth IMF represents the height of level flight, because the difference between the input data and the sum function of all IMFs without is large. The lack of the third IMF will lead to absolutely opposite trend on change of the cruising level which is fatal in trajectory prediction. The sum function without the second IMF leaded to more smoothing compared with the original height profile. It showed that the second IMF ensures the trajectory according with the real flight track. The first IMF cause overshoot when the aircraft firstly reaches the top of the trajectory, which can be realized as weather factor interfere with the level flight track of the aircraft.

\subsection{NOMINAL HEIGHT PROFILE PREDICTION}

Based on the IMFs according to the decomposition of flight height data recorded on various days, an optimization framework was built to calculate the fusion weight. For the day data, the corresponding IMFs are written as. For given the weight vector is defined as . To achieve the least square solution, optimization formulation is followed as where

For various flight day, the corresponding weight vector is calculated. And the IMF for flight height prediction is obtained by

Finally, the sum of weighted IMFs was realized as the predicted flight height profile. It was shown in Figure 3 with the other recorded historical height profiles. 
Figure 3. Predicted flight height profile based on proposed method when comparing with DWT method and historical data

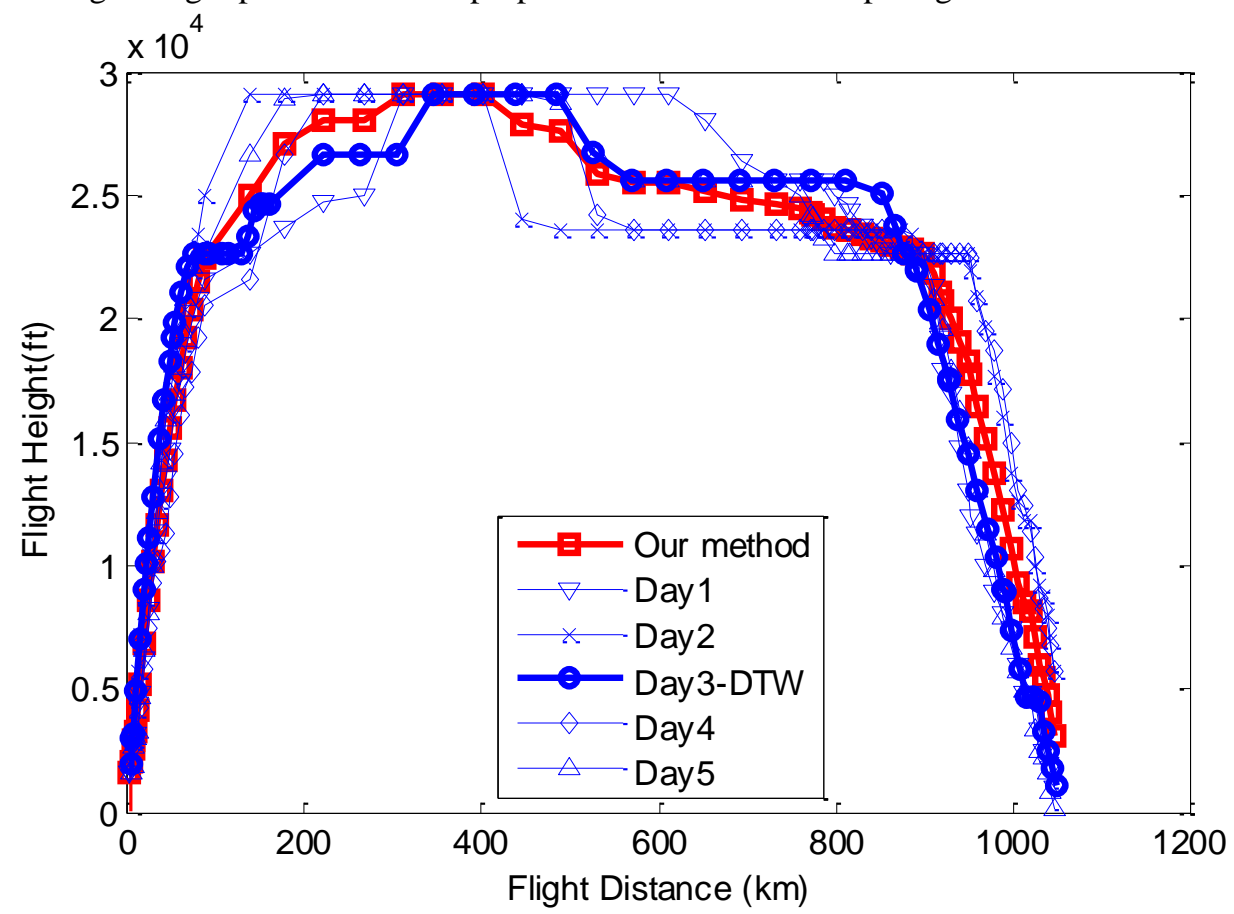

The nominal flight height profile obtained by nearest neighbor classification with dynamic time warping (DTW) was flight trajectory of the fourth day ${ }^{[\text {Tang2014] }}$. When comparing with this state-of-the-art method, a new nominal flight profile integrated several historical trajectories was obtained in he proposed method .

\section{CONCLUSION}

In this paper, an entirely data-driven flight height profile prediction approach is proposed. The historical flight data are firstly EMD. A similar procedure as leave-one-out cross validation (LOOCV) by calculating the NCC between the original data and sum functions of selected IMFs was applied to determine the represented flight intent for different IMFs. It is shown that the first IMF was realized as weather interference factor, while the second IMF represented the component that ensures the trajectory accord with the real flight track. The lack of the third and fourth IMFs leaded to large deviation on cruising level. The last IMF was with no clear physical meanings and causes no impact on flight height prediction. Finally, an optimization formulation was built to achieve regression weight under least square constraint. And a predicted flight height trajectory was obtained by sum these selected IMFs.

In the future, our proposed method will be extended to fuse the estimated flight profile with weather information to achieve 4D trajectory prediction with higher accuracy. It is able to solve the increasingly prominent contradiction between the rapid development of air transport industry and the limited resources of airspace. 
The authors gratefully acknowledge the financial support by Nanjing University of Aeronautics and Astronautics Innovation Fund Project (No. 56YAH14025), National Natural Science Foundation of China(61174180), Jiangsu Union Innovation Fund Projects (BY2012014), and Chinese Postdoctoral Science Foundation(2014M550291). 


\section{REFERENCES}

Castelli, L., Corolli, L., and Lulli, G. (2011). "Critical flights detected with time windows," Transportation Research Record, Journal Transportation Research Board, 2214: 103-110.

Flandrin, P., Rilling, G., and Goncalves, P., (2004). "Empirical mode decomposition as a filter bank," IEEE signal Processing Letter, 11:112-114.

Gariel, M., Srivastava, A. N., and E. Ferom, E. (2011). "Trajectory clustering and an application to airspace monitoring," IEEE Transations on Intelligent Transportation Systems, 12:1511-1524.

Huang, N. E., and Shen, S. S. P. (2005). Hilbert-Huang transform and its applications, World Scientific Pressing.

Huang, N. E., Shen, Z., and Long, S. R. (1999). "A new view of nonlinear water waves: the Hilbert spectrum," Annual Review Fluid Mechanical, 31:417-457.

Huang, N. E., Shen, Z., Long, S. R., and et al, (1998). "The empirical mode decomposition and the Hilbert spectrum for nonlinear and non-stationary time series analysis," Proceedings of Royal Society London: Series A, 454: 903-995.

Lv, X., (2007). “General framework of China's new generation civil aviation ATM system,” China Civil Aviation, 80(8):24-26.

Margellos, K., and Lygeros, J. (2013). "Toward 4D trajectory management in air traffic control: a study based on Monte Carlo simulation and reachability analysis," IEEE Transations on Control Systems Technology, 21: 1820-1833.

Matsuno, Y., and Tsuchiya, T., (2013). "4D trajectory optimization in the fresence of uncertainty," Proceedings of Aviation Technology, Integration, and Operations Conference, Los Angeles, CA, 1-11.

Shen, Z., Wang, Q., and Shen, Y. (2009). “A new non-liner correlation measure,” Proceedings of 2rd IEEE Youth Conference on Information, Computing and Telecommunication, Beijing, China, 2009, pp. $11-14$.

Tang, X.-M., Xing, J., Chen, P., and Shen, Z. (2014). "Generating nominal flight profile for air traffic control system based on AMARD data," Proceedings of IEEE $17^{\text {th }}$ International Conference on Intelligent Transportation System, Qindao, China, 2644-2649. 